\title{
Loci independent of mating type that condition secondary sexual incompatibility in the fungus Cochliobolus heterostrophus
}

\author{
James A. Kolmer and Kurt J. Leonard
}

\begin{abstract}
Department of Plant Pathology, Agricultural Research Service, United States Department of Agriculture, North Carolina State University, Raleigh, North Carolina 27695-7616.
\end{abstract}

\begin{abstract}
A full sib mating design was used to select for increased perithecial number in matings between sexually compatible pairs of ascospore progeny of the fungus Cochliobolus heterostrophus. In the third generation of selection approximately one-half of the isolate pairs produced abnormally low numbers of perithecia, indicating segregation at a single locus controlling fertility, which was independent of the mating type locus. We termed the phenomenon secondary sexual incompatibility, although it may also be related to vegetative compatibility. Large numbers of perithecia formed when the paired sexually compatible isolates had different alleles at the locus; few formed when they had identical alleles. Additional studies of two full sib families from another population showed the presence of at least four loci that condition secondary sexual incompatibility among these isolates. High fertility occurred only between isolates with different alleles at least one of the four loci. This phenomenon appears similar to the enhancement of fertility in Ceratocystis ulmi by genes for heterokaryon incompatibility, but the relationship of secondary sexual incompatibility loci to heterokaryon incompatibility loci in $\boldsymbol{C}$. heterostrophys has not been determined.
\end{abstract}

\section{INTRODUCTION}

Homogenic sexual incompatibility in fungi prevents self-fertilisation and sexual reproduction between isolates that have identical alleles at the mating type locus. Sexual incompatibility in basidiomycetes can be determined by as many as three loci, while in ascomycetes and oomycetes only one locus is usually involved (Burnett, 1975). Other loci, however, can also influence the degree of sexual compatibility in ascomycetes. For example, Nelson (1959) described a single defective gene in wild type isolates of Cochliobolus heterostrophus Drechsler that can eliminate or greatly reduce perithecial production when it is present in both members of a pair of sexually compatible isolates of the fungus. Kolmer and Leonard (1985) later showed that this gene or a similar gene acted additively. When both parents had the defective gene few perithecia were produced, but crosses in which only one parent had the defective gene produced an intermediate number of perithecia. Crosses in which neither parent

Paper number 10154 of the journal series of the North Carolina Agricultural Research Service, Raleigh 27695-7601. had the defective gene produced large numbers of perithecia. Webster and Nelson (1968) described two independent loci in $C$. spiciferus at which similar defective genes for perithecial production occur.

Kolmer and Leonard (1985) also showed that polygenic effects influenced the numbers of perithecia formed between sexually compatible isolates of $C$. heterostrophus. Selection for increased numbers of perithecia was carried out for six generations of intermatings among four to six parents in each generation which had been selected from approximately 24 progeny from the preceeding generation. After six generations the numbers of perithecia had increased by nearly a factor of four. The proportion of additive genetic variance present in the fungal population decreased linearly through the selection generations.

The present study was originally undertaken to compare different systems of selecting for increased perithecial numbers in $C$. heterostrophus. Our previously described selection utilised additive genetic variance (Kolmer and Leonard, 1985). In the present study, selection was based on epistatic genetic variance. We selected for perithecial number by inbreeding a population of the fungus 
for three generations using a full sib mating design. After three generations it became apparent that segregation for perithecial number was occurring at at least one locus that was independent of either the mating type locus (Nelson, 1957), or the defective perithecial gene locus (Nelson, 1959). In this paper we examine the genetic nature of this secondary incompatibility and estimate the numbers of loci that condition this trait in C. heterostrophus, the causal agent of southern corn leaf blight.

\section{MATERIALS AND METHODS}

The isolates used in this study were derived from an original population of 13 MATA and 12 MATa isolates, which have been previously described (Kolmer and Leonard, 1985). The 25 isolates were crossed in all sexually compatible combinations. Small blocks of agar with the fungus were cut from 4-day-old cultures on potato dextrose agar (PDA) containing $10 \mathrm{~g}$ dextrose per litre and were transferred to petri dishes containing modified Sachs agar (Hebert, 1971). Isolates of compatible mating types were placed opposite each other across autoclaved, $1 \mathrm{~cm}$. diam., senescent corn leaf discs on the modified Sachs agar. Four leaf discs were used for each MATA $\times M A T a$ combination. The isolate combinations were incubated in the dark at $25^{\circ} \mathrm{C}$ for 14 days. Mature perithecia that developed on the corn leaf discs were counted, with each leaf disc representing one replication for statistical purposes. All parental and progeny isolates were stored at $4^{\circ} \mathrm{C}$ in PDA slants.

\section{Full sib selection for perithecial number}

The MATA $\times$ MATa isolate combination with the highest average number of mature perithecia per leaf disc was selected as parents for the first generation of full sib selection. Ascospores were isolated from the selected cross after 21 days of incubation. Fertile perithecia were crushed in a drop of sterilised water on a sterile microscope slide and the contents were then washed onto an agar surface to separate the ascospores.

The full sib ascospore progeny were tested for mating type and then crossed in all sexually compatible combinations to evaluate the fertility of the first selection generation. The pair of progeny that produced the highest number of perithecia per leaf disc were chosen as parents for the next generation. This same full sib mating design and selection for increased numbers of perithecia continued through three generations. Approximately 10 isolates of each mating type were used in producing perithecia in each selection generation. The components of genetic variation in the initial and selection generations were partitioned as previously described (Kolmer and Leonard, 1985). The effective perithecial allele (Nelson, 1959) was not a source of variation since it was fixed in the first generation.

\section{Pedigrees of isolates}

The pedigrees of the ascospore families used in the study of the secondary sexual incompatibility $(S S I)$ loci in $C$. heterostrophus are listed in table 1. The ascospore isolates in this part of the study were maintained and crossed in the same manner as described above. Crosses between sexually compatible isolates that produced abnormally low numbers of mature perithecia were scored as secondary sexually incompatible; crosses that produced large numbers of mature perithecia were scored as secondary sexually compatible.

Table 1 Pedigrees of Cochliobolus heterostrophus full sib families used in the study of secondary sexual incompatibility

\begin{tabular}{|c|c|}
\hline $\begin{array}{l}\text { Family } \\
\text { designation }\end{array}$ & Origin \\
\hline Full Sib (FS) 1 & $\begin{array}{l}\text { Derived from } 3 \text { generations of full sib } \\
\text { selection for perithecial number }\end{array}$ \\
\hline FS 1A & $\begin{array}{l}\text { Derived from the cross of isolates FS } 1-1 \\
M A T A \times \text { FS } 1-4 M A T a \text { from FS } 1\end{array}$ \\
\hline FS 1B & $\begin{array}{l}\text { Derived from a cross of isolates FS } 1-2 \\
M A T A \times \text { FS } 1-3 M A T a \text { from FS } 1\end{array}$ \\
\hline FS $1 \mathrm{C}$ & $\begin{array}{l}\text { Derived from a cross of isolates FS } 1-1 \\
M A T A \times \text { FS } 1-3 M A T a \text { from FS } 1\end{array}$ \\
\hline FS 2 & $\begin{array}{l}\text { Derived from a cross of } M A T A \times M A T a \\
\text { isolates taken from the } 5 \text { th generation of } \\
\text { general selection for perithecial number }\end{array}$ \\
\hline FS 3 & $\begin{array}{l}\text { Derived from the cross of } M A T A \times M A T a \\
\text { isolates taken from the } 6 \text { th generation of } \\
\text { selection for perithecial number }\end{array}$ \\
\hline
\end{tabular}

\section{RESULTS}

\section{Selection for perithecial number}

Selection with a full sib mating design resulted in an increase in the mean number of perithecia per leaf disc through the first two generations, but the number declined in the third generation (table 2). With the general selection design, the mean numbers of perithecia continued to increase, so that many more perithecia were produced in the third selection generation when compared with selection using a full sib mating design. The proportion of 
Table 2 Changes in mean number of perithecia per leaf disc and proportion of additive genetic variance through three generations of full sib selection and general selection for increased numbers of perithecia in Cochliobolus heterostrophus

\begin{tabular}{|c|c|c|c|c|}
\hline Generation & $\begin{array}{l}\text { Mean number } \\
\text { perithecia } \\
\text { full sib } \\
\text { selection }\end{array}$ & $\begin{array}{l}\text { Mean number } \\
\text { perithecia } \\
\text { general } \\
\text { selection* }\end{array}$ & $\begin{array}{l}\text { Proportion } \\
\text { additive } \\
\text { genetic } \\
\text { variance } \\
\text { full sib } \\
\text { selection }\end{array}$ & $\begin{array}{l}\text { Proportion } \\
\text { additive } \\
\text { genetic } \\
\text { variance } \\
\text { general } \\
\text { selection* }\end{array}$ \\
\hline 0 & 44 & 44 & 0.83 & 0.83 \\
\hline 1 & 84 & 90 & $0 \cdot 31$ & 0.72 \\
\hline 2 & 85 & 71 & $0 \cdot 17$ & 0.66 \\
\hline 3 & 62 & 100 & 0.00 & 0.53 \\
\hline
\end{tabular}

* Kolmer and Leonard, 1985

additive genetic variance declined rapidly and was reduced to zero by the third generation of full sib selection. With general selection the proportion of additive genetic variance declined less rapidly and at a linear rate over six generations (Kolmer and Leonard, 1985).

\section{Segregation for secondary sexual incompatibility}

In full sib family \# 1 (FS 1), which constituted the third generation of full sib selection, the mated pairs segregated into two distinct classes with respect to numbers of perithecia produced. Approximately half the pairings between sexually compatible isolates produced fewer than a mean of 30 perithecia per leaf disc. In these pairings the perithecia formed only along the center axis of the leaf disc, where the mycelia of the paired isolates first met. The other half of the pairings of sexually compatible isolates produced a mean of nearly 100 perithecia per leaf disc (table 3 ). Perithecia in these crosses formed over the entire surface of the leaf disc. It was apparent that the isolates in both mating types were segregating at a single locus which conditioned, what we have termed secondary sexual incompatibility $(S S I)$. We hypothesised that perithecial number was low when paired sexually compatible isolates had similar alleles at the SSI locus and high when different alleles were present.

To test the hypothesis further, three sets of full sib ascospore progeny FS $1 \mathrm{~A}$, FS $1 \mathrm{~B}$, and FS $1 \mathrm{C}$, were obtained from the crosses of two MATA and two MATa isolates in FS 1 (table 1). FS $1 \mathrm{~A}$ and FS 1B were derived from matings that produced very low numbers of perithecia per leaf disc. The parent isolates of FS $1 \mathrm{C}$ produced a large number of perithecia per leaf disc when paired together.
Ascospore isolates from FS $1 \mathrm{~A}$, and FS 1B, when mated in full sib sexually compatible combinations were secondary sexually incompatible, producing only a low number of perithecia per leaf disc (table 3 ). Ascospore isolates from FS 1C when mated in sexually compatible pairs segregated at the $S S I$ locus in a manner similar to FS 1 (table 3).

Isolates in FS 1A were mated with isolates in FS $1 B$ in all sexually compatible combinations. All 12 pairs of isolates were secondary sexually compatible, producing large numbers of perithecia. Isolates in FS 1A were also mated with isolates in FS $1 \mathrm{C}$ in all sexually compatible combinations. Of the 78 isolate pairs, 30 were secondary sexually compatible, and 48 were secondary sexually incompatible. Isolates in FS $1 \mathrm{~A}$ did not segregate for level of fertility with isolates of compatible mating type from FS $1 \mathrm{C}$; they were all highly fertile or weakly fertile with a given isolate. Isolates from FS 1C, however did segregate approximately $1: 1$ for level of fertility with FS 1A isolates (table 3 ).

Table 3 Segregation of SSI and MAT alleles and effects of secondary sexual incompatibility on numbers of perithecia produced by isolates of Cochliobolus heterostrophus derived from full sib family 1

\begin{tabular}{|c|c|c|c|}
\hline Family & MATA MATa & $\begin{array}{l}\text { Number of } \\
\text { combinations }\end{array}$ & $\begin{array}{l}\text { Perithecia/ } \\
\text { leaf disc }\end{array}$ \\
\hline \multirow{3}{*}{ FS 1} & $\mathrm{SSI} \times \mathrm{SSI}$ & $5 \times 5$ & $24 \cdot 4 \pm 1.97$ \\
\hline & $\begin{array}{l}\text { SSI } \times \text { ssi } \\
\text { ssi } \times \text { SSI }\end{array}$ & $\begin{array}{l}5 \times 3 \\
5 \times 5\end{array}$ & $97 \cdot 0 \pm 1 \cdot 53$ \\
\hline & ssi $\times$ ssi & $5 \times 3$ & $25 \cdot 8 \pm 2 \cdot 76$ \\
\hline FS $1 A$ & $\mathrm{SSI} \times \mathrm{SSI}$ & $6 \times 3$ & $26 \cdot 0 \pm 0 \cdot 50$ \\
\hline FS 1B & ssi $\times s s i$ & $2 \times 1$ & $23 \cdot 5 \pm 1 \cdot 5$ \\
\hline \multirow{3}{*}{ FS $1 \mathrm{C}$} & $\mathrm{SSI} \times \mathrm{SSI}$ & $4 \times 6$ & $21 \cdot 6 \pm 1 \cdot 73$ \\
\hline & $\begin{array}{l}\text { SSI } \times \text { ssi } \\
s s i \times S S I\end{array}$ & $\begin{array}{l}4 \times 4 \\
2 \times 6\end{array}$ & $81 \cdot 1 \pm 2 \cdot 63$ \\
\hline & ssi $\times$ ssi & $2 \times 4$ & $24 \cdot 8 \pm 1 \cdot 43$ \\
\hline
\end{tabular}


Identification of secondary sexual incompatibility loci

To determine if additional loci in C. heterostrophus condition secondary sexual incompatibility, ascospore families FS 2 and FS 3 (table 1) were obtained from the fifth and sixth generations of a genetically disparate population of $C$. heterostrophus which had been selected for increased perithecial number in a general selection design (Kolmer and Leonard, 1985). Families FS 2 and FS 3 segregated at a single $S S I$ locus when isolates in each family were intermated in a full sib design. Crosses between isolates of FS 2 and FS 3 were all secondary sexually compatible, producing a large number of perithecia in all pairs.

Four sets of ascospore isolates were obtained from crosses of two FS 2 MATA isolates with two FS $3 M A T a$ isolates. The four families of ascospore progeny were then crossed to sexually compatible tester isolates. The set of tester isolates was composed of the four parental isolates and isolates from FS 2 and FS 3 with equivalent secondary sexual incompatibitility reaction but of opposite mating type from the parent isolates (table 4).

Progeny from FS 2-1 MATA $\times$ FS 3-1 MATa and FS 2-2 MATA $\times$ FS 3-2 MATa segregated for the two parental classes and two recombinant types when evaluated with the tester isolates, indicating segregation at two SSI loci. Progeny from FS 2-1 $M A T A \times$ FS 3-2 MATa and FS 2-2 MATA $\times$ FS
3-1 MATa segregated only for the two parental classes, indicating segregation at a single $S S I$ locus (table 4).

Isolates from FS 1 were secondary sexually compatible when mated with opposite mating type isolates from FS 2 and FS 3. Progeny from all four families from FS $2 M A T A \times$ FS 3 MATa were secondary sexually compatible when mated with opposite mating type isolates FS 1-1 or FS 1-2, or isolates from FS 1 that were equivalent to FS 1-1 or FS 1-2 except at the mating type locus. This indicated that isolates in FS 1 were fixed for a different allele than FS 2 and FS 3 at at least one SSI locus.

\section{Determination of secondary sexual incompatibility genotypes}

In order to determine the genotypes of isolates in FS 1 relative to isolates in FS 2 and FS 3, isolate FS 2-2 MATA was crossed with isolates equivalent with FS 1-2 MATA and FS 1-1 MATa except at the mating type locus (FS 1-2 MATa, FS 1-1 $M A T a)$. The progeny were mated to the tester isolates listed in table 4 and isolates FS 1-1 and FS 1-2 or isolates from FS 1 that were equivalent to FS 1-1 and FS 1-2 except at the mating type locus. Progeny from FS 2-2 MATA × FS 1-1 MATa segregated for the parental types, a recombinant type identical to a tester isolate (FS 2-1), and isolates that were compatible with all six testers.

Table 4 Segregation of progeny isolates from FS 2 crossed with FS 3 of Cochliobolus heterostrophus for secondary incompatibility as determined by four tester genotypes*

\begin{tabular}{|c|c|c|c|c|c|c|}
\hline \multirow{2}{*}{$\begin{array}{l}\text { Isolates } \\
\text { crossed }\end{array}$} & \multirow{2}{*}{$\begin{array}{l}\text { Number } \\
\text { of } \\
\text { progeny }\end{array}$} & \multicolumn{4}{|c|}{ Tester isolates } & \multirow{2}{*}{$\begin{array}{l}\text { Expected } \\
\text { seg. }\end{array}$} \\
\hline & & FS 2-1 & FS 2-2 & FS 3-1 & FS 3-2 & \\
\hline FS 2-1 MATA & 5 & $t^{*}$ & + & $-\dagger$ & + & 1 \\
\hline$\times$ & 3 & + & - & + & + & 1 \\
\hline \multirow[t]{2}{*}{ FS 3-1 MATa } & 7 & - & + & + & + & 1 \\
\hline & 4 & + & + & + & - & 1 \\
\hline $\begin{array}{c}\text { FS 2-1 MATA } \\
\times\end{array}$ & 3 & - & + & + & + & 1 \\
\hline \multirow{2}{*}{$\begin{array}{l}\text { FS } 3-2 \text { MATa } \\
\text { FS } 2-2 \text { MATA }\end{array}$} & 6 & + & + & + & - & 1 \\
\hline & 9 & + & + & - & + & 1 \\
\hline \multirow{2}{*}{$\begin{array}{l}\text { FS } 3-1 \text { MATa } \\
\text { FS } 2-2 \text { MATA }\end{array}$} & 7 & + & - & + & + & 1 \\
\hline & 3 & + & + & + & - & 1 \\
\hline \multirow{3}{*}{ FS $\stackrel{\times}{3-2} M A T a$} & 5 & - & + & + & + & 1 \\
\hline & 4 & + & - & + & + & 1 \\
\hline & 6 & + & + & - & + & 1 \\
\hline
\end{tabular}

\footnotetext{
* Each secondary incompatibility tester genotype was present in both MATA and MATa mating types.

$\dagger+$ Indicates large numbers of perithecia produced; - indicates few perithecia. See Table 3 for relative numbers.
} 
Progeny from FS 2-2 MATA $\times$ FS 1-2 MATa segregated for one parental type, two recombinant types identical to tester isolates (FS 2-1, FS 3-2), and isolates that were compatible with all tester isolates (table 5).

Table 5 Segregation of progeny from the matings of isolates in FS $2 \times$ isolates in FS 1 of Cochliobolus heterostrophus for secondary incompatibility as determined by six tester genotypes*

\begin{tabular}{lll}
\hline & $\begin{array}{l}\text { Number of } \\
\text { progeny }\end{array}$ & SSI type \\
\hline $\begin{array}{c}\text { FS 2-2 MATA } \\
\times\end{array}$ & 1 & FS 2-2 \\
FS 1-1 MATA & 4 & FS 1-1 \\
& 16 & FS 2-1 \\
FS 2-2 MATA & 1 & High fertility with \\
$\times$ & 0 & all testers \\
FS 1-2 MATa & 1 & FS 2-2 \\
& 3 & FS 2-1 \\
& 17 & FS 3-2 \\
& & High fertility with \\
\hline
\end{tabular}

* Each secondary incompatibility tester genotype was present in both MATA and MATa mating types.

${ }^{b}$ Isolates in the same $S S I$ group are only weakly fertile in matings between MATA and MATa mating types. Isolates from different $S S I$ groups are highly fertile.

To further determine the number of genotypes present in the progeny of the two families, the 17 isolates from FS 2-2 MATA $\times$ FS 1-2 MATa, and the 16 isolates from FS 2-2 MATA $\times$ FS 1-1 MATa that had high fertility with all the tester isolates, were mated in full sib combinations according to mating type. These isolates were also mated in half sib combinations in crosses between the two families. From the patterns of secondary sexual incompatibility and compatibility in these crosses, the progeny could be sorted into additional classes of genotypes (fig. 1). The progeny from both families segregated into at least six additional genotypes. A total of at least nine and a maximum of 10 secondary sexual incompatibility genotypes segregated in the cross of FS 2-2 MATA $\times$ FS 1-1 $M A T a$. A total of at least nine, and a maximum of 13 secondary sexual incompatibility genotypes segregated in the cross of FS 2-2 MATA $\times$ FS 1-2 $M A T a$ (fig. 1). This indicated that progeny from crosses of isolate FS 2-2 MATA with FS 1-1 MATa and FS 1-2 MATa segregated for at least four loci that condition secondary sexual incompatibility.

From the above crosses and segregations, we were able to determine the genotypes of isolates in FS 1, FS 2, and FS 3 at four SSI loci (table 6).

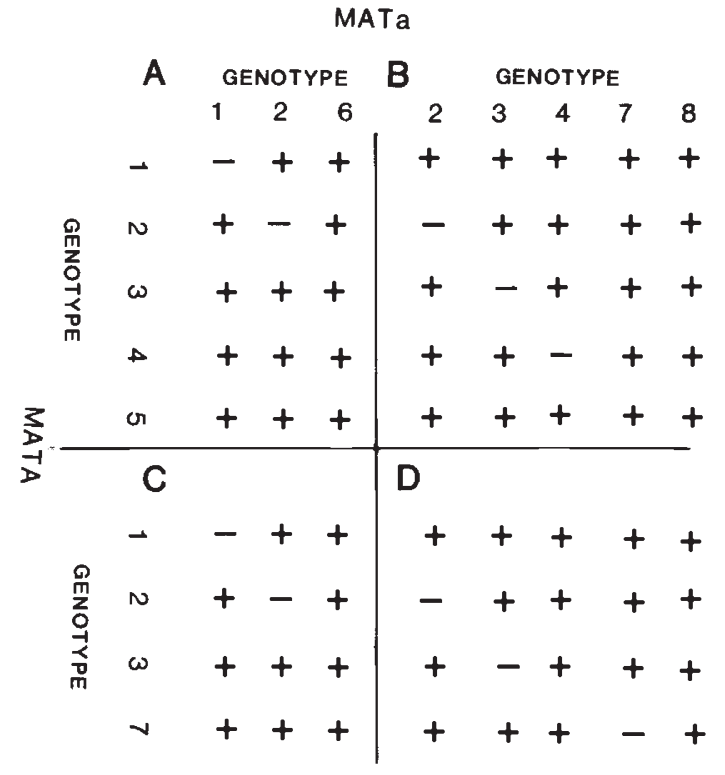

Figure 1 Full sib and half sib crosses of progeny from FS 2-2 MATA $\times$ FS 1-1 MATa and FS 2-2 MATA $\times$ FS 1-2 MATa, $(+)$ indicates large numbers of perithecia formed, $(-)$ indicates few perithecia formed. One representative isolate of each genotype from both crosses is shown. Four isolates were geneotype 6, two isolates were genotype 8 . (A) FS $2-2$ MATA $\times$ FS 1-1 MATa progeny mated in full sib combinations. (B) FS 2-2 $\times$ FS 1-1 MATA progeny crossed with FS 2-2 $\times$ FS 1-2 MATa progeny in half sib combinations. (C) FS 2-2 $\times$ FS 1-1 MATa progeny crossed with FS $2-2 \times$ FS 1-2 MATA progeny in half sib combinations. (D) FS $2-2 \times$ FS 1-2 progeny mated in full sib combinations.

Table 6 Proposed genotypes of Cochliobolus heterostrophus isolates in FS 1, FS 2, and FS 3, at four SSI loci

\begin{tabular}{ll}
\hline Isolates & $S S I$ genotypes \\
\hline FS 1-1 & $S S I 1-1, S S I 2-1, S S I 3-2, S S I 4-2$ \\
FS 1-2 & $S S I 1-1, S S I 2-2, S S I 3-2, S S I 4-2$ \\
FS 2-1 & $S S I 1-1, S S I 2-1, S S I 3-1, S S I 4-1$ \\
FS 2-2 & $S S I 1-2, S S I 2-1, S S I 3-1, S S I 4-1$ \\
FS 3-1 & $S S I 1-2, S S I 2-2, S S I 3-1, S S I 4-1$ \\
FS 3-2 & $S S I 1-1, S S I 2-2, S S I 3-1, S S I 4-1$ \\
\hline
\end{tabular}

\section{DISCUSSION}

The patterns of relative perithecial number in crosses among isolates derived from three full sib families of $C$. heterostrophus consistently showed segregation at loci, independent of the mating type locus, that condition the relative numbers of perithecia formed per cross. These loci differ from the perithecial gene locus (Nelson, 1959) and apparently are not closely linked to it, since the parental isolates of the three full sib families were fixed for the effective gene. 
Selection for increased perithecial number using a full sib mating design resulted in lower levels of fertility and a rapid decline in the proportion of additive genetic variance when compared with the general selection for fertility. This confirms that inbreeding occurred much more rapidly with full sib selection, and that full sib selection, which is based on specific interactions between isolates, is less effective than selection based on additive genetic vairance.

The segregation patterns for relative perithecial numbers obtained from FS $1 \mathrm{~A}, \mathrm{FS} 1 \mathrm{~B}$, and FS $1 \mathrm{C}$, show that perithecial number is high in crosses between sexually compatible isolates that have different alleles at at least one $S S I$ locus. All mating type compatible crosses between FS $1 \mathrm{~A}$ and FS 1B produced high numbers of perithecia, confirming that the presence of unlike alleles at an SSI locus conditioned high fertility. Since the parents of FS $1 \mathrm{C}$ produced a large number of perithecia when mated, we correctly predicted that the progeny would segregate at one locus for perithecial number. The segregations for perithecial number from crosses between isolates in FS $1 \mathrm{~A}$ and FS $1 \mathrm{C}$ also supported the conclusion that different alleles at an $S S I$ locus condition large numbers of perithecia in crosses between MATA and MATa isolates.

Segregation at a single $S S I$ locus was found in FS 1 only after three generations of inbreeding, which indicates that single alleles had been fixed at other $S S I$ loci in the preceeding generations of inbreeding. None of the 100 MATA $\times$ MATa combinations in the first generation of full sib selection produced abnormally low numbers of perithecia, while in the second generation, five of the 75 isolate pairs produced few perithecia relative to the other pairs. Apparently, fixation at an SSI locus first occurred in the second generation of selection, and by the third generation all but one locus had been fixed for single alleles.

Progeny from FS $2 \times$ FS 3 segregated at only two SSI loci, which indicated that the two families had become fixed for identical alleles at other SSI loci during the general selection for increased perithecial number. In the fifth and sixth generations of general selection nine of the 120 isolate pairs and 14 of the 84 pairs, respectively, were secondary sexually incompatible, and produced abnormally low numbers of perithecia. The increased frequency of secondary sexually incompatible isolate pairs reflects the gradual fixation of alleles at the $S S I$ loci during the selection process.

The epistatic effect of one SSI locus with different alleles to other loci that have identical alleles is seen in the segregation of progeny from
FS 2-1 MATA $\times$ FS 3-1 MATa and FS 2-2 MATA $\times$ FS 3-2 MATa. Nonparental progeny share identical alleles with one or the other parental type at at least one locus; however, they have different alleles at one other SSI locus. Thus, these isolate pairs are secondary sexually compatible.

The progeny of FS 2-2 MATA $\times$ FS 1-1 MATa, and FS 2-2 MATA $\times$ FS 1-2 MATa, segregated into a large number of SSI types indicating that isolates in FS 2 and FS 3, have different alleles at at least three SSI loci than isolates in FS 1. This is not surprising since FS 2 and FS 3 are quite genetically disparate from isolates in FS 1 . We estimate the total number of SSI loci to be at least four.

The presence of SSI loci promotes outbreeding among closely related, sexually compatible isolates of $C$. heterostrophus. As the number of SSI loci increases, however, their effectiveness decreases. Segregation at one $S S I$ locus reduces fertility by approximately 50 per cent in matings between sexually compatible full sib isolates, while segregation at two loci reduces fertility by only 25 per cent.

The $S S I$ loci in $C$. heterostrophus may be related to heterogenic vegetative incompatibility previously described in the fungus (Leach and Yoder, 1983). Leach and Yoder (1983) did not identify specific loci for vegetative incompatibility nor determine the number of such loci in $C$. heterostrophus, but their evidence indicated that multiple loci are involved. Many ascomycetes are known to have large numbers of vegetative compatibility groups. Successful anastomosis of vegetative hyphae occurs only between isolates within the same vegetative compatibility group, i.e., between isolates with identical alleles at all of the vegetative compatibility loci (Esser and Blaich, 1973).

In Ceratocysits ulmi the genes for vegetative incompatibility have been shown to have a major effect on the numbers of perithecia produced in matings of sexually compatible isolates (Brasier, 1984). In combinations of vegetatively compatible isolates of opposite mating type, perithecia formed only along the junction line in the area of immediate mycelial confrontation of mycelia of the two isolates. In sexual crosses of vegetatively incompatible isolates, however, mycelia penetrated beyond the junction line, and perithecia formed over much of the mycelial area of both isolates.

Since the vegetative incompatibility genes do not disrupt plasmogamy and karyogamy in sexual reproduction, the vegetative incompatibility system must be suppressed or bypassed in interactions between isolates of opposite mating type. Rayner, et al., (1984) have proposed an override system, based on non-self recognition that suppresses the 
rejection response between unlike genotypes when they are sexually compatible. In ascomycetes this mighi involve suppression of vegetative incompatibility in cells differentiated as ascogonia, or products of mating type complementation may block the normal vegetative incompatibility response (Brasier, 1984).

There are striking parallels between the pattern of fertility levels in matings among SSI genotypes of $C$. heterostrophus and the demonstrated involvement of vegetative incompatibility genes in determining fertility levels in C. ulmi. It may be tempting to equate the SSI loci with loci for vegetative incompatibility in C. heterostrophus, but this would be premature, because little is known of vegetative incompatibility loci in C. heterostrophus. Leach and Yoder (1983) did not observe barrage formation or other morphological responses to distinguish vegetatively incompatible interactions in this fungus. Demonstratiori of vegetative incompatibility by inducing auxotrophic mutants in wild type isolates and pairing them on minimal media is a laborious process, and is poorly suited for surveying vegetative compatibility groups among natural populations. Furthermore, none of the auxotrophic mutants that Leach and Yoder (1983) worked with retained the sexual fertility of their wild type parent isolates. Therefore, genetic analysis of vegetative incompatibility loci and their relationship to $S S I$ loci would be difficult.

A high degree of polymorphism affecting fertility has been found in populations of $C$. heterostrophus. We found segregation at at least four SSI loci, and earlier (Kolmer and Leonard, 1985) found extensive quantitative genetic variation affecting numbers of perithecia produced in culture. Nelson (1959) also found different alleles at the perithecial gene locus in field populations of the fungus. Thus, numbers of perithecia produced in crosses of MATA $\times$ MATa isolates of C. heterostropus are determined by a complex of different genetic systems, which include Mendelian loci and polygenic effects. It would appear, however, that the ability of an isolate to produce perithecia has little or no relation to its fitness in nature. Isolates of the fungus that have been selected for high fertility produced lesions on corn plants that did not differ significantly in size from those of original parental isolates (Kolmer and Leonard, 1986).

In general, characters with low heritabilities are more closely connected with reproductive fitness than characters with the highest heritabilities (Falconer, 1980). Recurrent selection for virulence in a population of $C$. heterostrophus resulted in selected isolates that produced lesions only slightly, although significantly, longer than those of the parental isolates on resistant corn inbreds (Kolmer and Leonard, 1986). The realized heritability estimate for lesion length in $C$. heterostrophus was $0 \cdot 27$, compared to an estimate of 0.74 for polygenic effects influencing perithecial production (Kolmer and Leonard, 1985).

The $S S I$ loci in $C$. heterostrophus can serve as valuable markers in determining polymorphisms in field populations of the fungus. We are currently comparing populations of race $\mathrm{T}$ of the fungus from 1970 and 1973 (Leonard, 1973) for polymorphisms at the SSI loci, this may help to determine the origin of race $T$ in relation to its sudden spread in the 1970 southern corn leaf blight epidemic in the United States.

\section{REFERENCES}

BRASIER, C. M. 1984. Intermycelial recognition systems in Ceratocystic ulmi: Their physiological properties and ecological importance. D. H. Jennings and A. D. M. Rayner (eds) Cambridge University Press, Cambridge, pp. 451497.

BURNetT, J. H. 1975. Mycogenetics. John Wiley and Sons, London.

ESSER, K. AND BLAICH, R. 1973. Heterogenic incompatibility in plants and animals. Advances in Genetics, 17, 107-152.

FALCONER, D. S. 1980. Introduction to Quantitative Genetics. Longham, New York.

HEBERT, T. T. 1971. The perfect stage of Pyricularia grisea. Phytopathology, 61, 83-87.

KOLMER, J. A. AND LEONARD, K. J. 1985. Genetic variation and selection for fertility in the fungus Cochliobolus heterostrophus. Heredity, 55, 335-339.

KOLMER, J. A. AND LEONARD, K. J. 1986. Genetic selection and adptation of Cochliobolus heterostrophus to corn hosts with partial resistance. Phytopathology, 76, In press.

LEACH, J. AND YODER, O. C. 1983. Heterokaryon incompatibility in the plant pathogenic fungus Cochiobolus heterostrophus. J. of Heredity, 74, 149-152.

LEONARD, K. J. 1973. Association of mating type and virulence in Helminthosporium maydis, and observations on the origin of the race $T$ population in the United States. Phytopathology, 63, 112-115.

NELSON, R. R. 1959. Genetics of Cochliobolus heterostrophus IV. A mutant gene that prevents perithecial formation. Phytopathology, 49, 384-386.

RAYNER, A. D. M., COATES, D., AINSWORTH, A. M., ADAMS, T. J. H., WILliAMS, E. N. D. AND TODD, N. K. 1984. The biological consequences of the individualistic mycelium. D. H. Jennings and A. D. M. Rayner (eds) In Ecology and Physiology of the Fungal Mycelium. Cambridge Universtity Press, Cambridge, pp. 509-540.

WEBSTER, R. K. AND NELSON, R. R. 1968. The genetics of Cochliobolus speciferus. I. Genetic inhibition of perithecial and ascus formation. Can. J. Bot. 46, 197-202. 